\title{
ULTRAPERIPHERAL PHOTOPRODUCTION OF VECTOR MESONS IN THE NUCLEAR COULOMB FIELD AND THE SIZE OF NEUTRAL VECTOR MESONS
}

\author{
S.R. GEVORKYAN ${ }^{1,2}$, I.P. IVANOV ${ }^{3,4}$ AND N.N. NIKOLAEV ${ }^{3,5}$ \\ ${ }^{1}$ Joint Institute of Nuclear Research,141980 Dubna,Russia \\ 2 Yerevan Physics Institute, 375036, Yerevan, Armenia \\ 3 IKP, Forschungszentrum Jülich,D-52425 Jülich, Germany \\ ${ }^{4}$ Sobolev Institute of Mathematics, 630090, Novosibirsk, Russia \\ ${ }^{5}$ L. D. Landau Institute for Theoretical Physics, 142432 Chernogolovka, \\ Russia
}

\begin{abstract}
We point out a significance of ultraperipheral photoproduction of vector mesons in the Coulomb field of nuclei as a means of measuring the radius of the neutral vector meson. This new contribution to the production amplitude is very small compared to the conventional diffractive amplitude, but because of large impact parameters inherent to the ultraperipheral Coulomb mechanism its impact on the diffraction slope is substantial. We predict appreciable and strongly energy dependent increase of the diffraction slope towards very small momentum transfer. The magnitude of the effect is proportional to the mean radius squared of the vector meson and is within the reach of high precision photoproduction experiments, which gives a unique experimental handle on the size of vector mesons.
\end{abstract}

\section{Introduction}

It was noticed long time ago [1], that photoproduction of pseudoscalar mesons in the Coulomb field of nuclei allows one to measure the radiative width of mesons $\left(\pi^{0} \rightarrow \gamma \gamma, V \rightarrow P \gamma\right.$ etc.,for the review and references see [2]). The principal point is that the Primakoff amplitude can be isolated for the presence of the Coulomb pole in the production amplitude, so that the contribution to the differential cross section of the process $\gamma+A \rightarrow$ 
$P+A, P=\left(\pi^{0}, \eta, \eta^{\prime}\right)$ from one-photon Coulomb exchange reads

$$
\frac{d \sigma}{d q^{2}}=\frac{8 \pi \alpha_{e m} Z^{2}}{m_{P}{ }^{3}} \Gamma_{P} \frac{q^{2}}{\left(q^{2}+\Delta^{2}\right)^{2}} F_{A}^{2}(\vec{q}) .
$$

Here $m_{P}$ is the mass of the meson, $Z$ is the nucleus charge number; $\alpha_{e m}=$ $\frac{e^{2}}{4 \pi}$ is the fine structure constant, $q=|\vec{q}|$ and

$$
\Delta=\frac{m_{P}^{2}}{2 E_{\gamma}}
$$

are the transverse and the longitudinal momentum transfer, respectively, $F_{A}(\vec{q})$ is the nuclear charge form factor and $\Gamma_{P}$ is the two-photon decay width of the pseudoscalar meson. The differential cross section (1) peaks at $\vec{q}^{2}=\Delta^{2}$, where it rises with the photon's energy $E_{\gamma}$ as $\frac{d \sigma}{d t} \propto 1 / \Delta^{2} \propto E_{\gamma}^{2}$, whereas the position of the peak shifts to lower values of $\vec{q}^{2} \propto 1 / E_{\gamma}^{2}$. This property allows one to isolate the Coulomb contribution unambiguously and measure the two-photon decay width $\Gamma_{P}=\Gamma(P \rightarrow 2 \gamma)$ of pseudoscalar mesons. The recent progress of the experimental technique and high intensity photon beams available at CEBAF have lead to a new proposal of the measurement of the neutral pion lifetime at the level of several per mill, which would allow crucial tests of the chiral anomaly [3].

Subsequently, Pomeranchuk and Shmushkevich [4] extended the method to the determination of the lifetime of the $\Sigma^{0}$-hyperon via Coulomb production of the $\Sigma^{0}$ in the beam of $\Lambda^{0}$-hyperons. Subsequently, many radiative width of many charged meson resonances have been measured via Coulomb photoproduction of resonances in the pion and kaon beams (for the review and references see [2] and the Review of Particle Properties [6]). Because of the C-parity constraints, the Primakoff effect does not contribute to the photoproduction of the vector mesons, and one needs at least two-photon exchange. The two-photon exchange contribution to the photoproduction amplitude will no longer contain the Coulomb pole inherent to the Primakoff contribution. However, the principal point that in the Coulomb amplitude the important impact parameters are very large,

$$
|\vec{b}| \sim \frac{1}{\Delta}
$$

remains very much relevant. Furthermore, according to (2), the range of relevant impact parameters rises rapidly with energy. The Coulomb contribution to the photoproduction amplitude from these large impact parameters has logarithmic singularity $\propto \log \left[1 / R_{A}^{2}\left(\vec{q}^{2}+\Delta^{2}\right)\right]$, which generates the singular dependence of the forward diffraction slope on $\vec{q}^{2}$ and energy.Finally, invoking the familiar vector meson dominance, the Coulomb contribution 
to the photoproduction amplitude can be related to the amplitude of elastic scattering of the vector meson in the Coulomb field. To the two-photon exchange approximation, the latter is proportional to the radius squared of the vector meson. Consequently, the experimental isolation of the ultraperipheral Coulomb contribution to the diffractive vector meson production would lead to a unique experimental measurement of the size of neutral vector mesons and must not be overlooked.

The estimations of higher order Coulomb corrections have been performed earlier for photoproduction of pseudoscalar mesons [5]. There, too, multiple Coulomb exchanges have the logarithmic singularity, but they are too weak to produce a numerically substantial correction to the Primakoff amplitude with the pole singularity.

\section{Color and electric dipole view at the ultraperipheral Coulomb production and the radius of vector mesons}

Because of the rise of the coherence and formation times, at high energies the meson photoproduction can be viewed as a three step process: splitting of the photon into quark-antiquark pair at a large distance in front of the target, interaction of the quark-antiquark color and electric dipole with the target, and projection of the scattered quark-antiquark pair onto the observed meson. When such a target is a Coulomb field of heavy nuclei, it is not á priori obvious that higher order Coulomb corrections will be small, because the QED expansion parameter $Z \alpha_{e m} \sim 1$. However, because mesons are electrically neutral and have a small size $R_{M}$, the strength of the interaction of small electric dipole at large impact parameters $\vec{b}$ is suppressed by the small parameter $R_{M}^{2} / \vec{b}^{2}$, so that the ultraperipheral Coulomb production can be estimated to the leading two-photon exchange approximation.

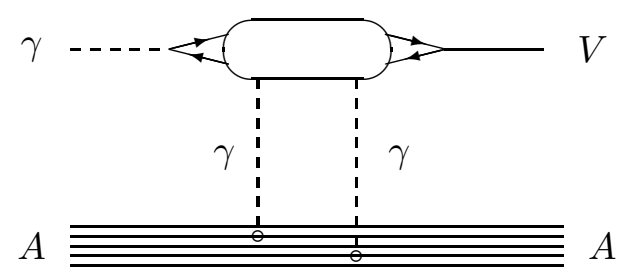

Figure 1. The two-photon exchange contribution to the photoproduction amplitude

The technical argument why the Coulomb contribution is singular and measures the size of the vector meson goes as follows. The vector meson 


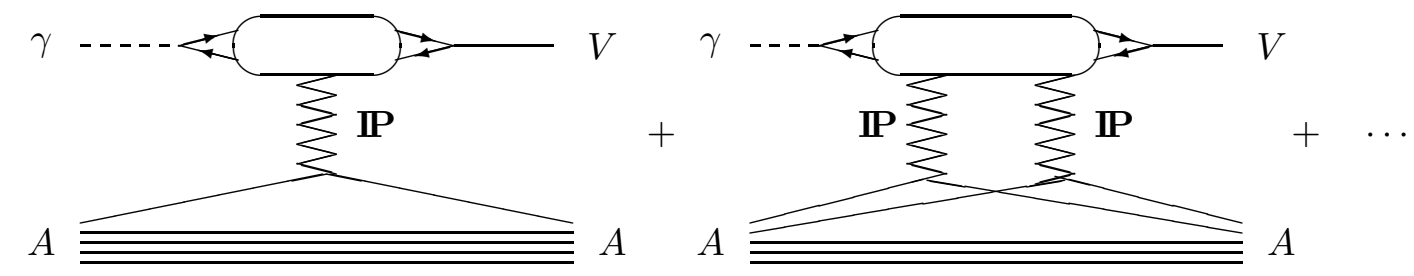

Figure 2. Left: the single-pomeron exchange, i.e., impulse approximation, contribution to the photoproduction off a nucleus,right: the multiple exchange contribution to the the photoproduction off a nucleus.

photoproduction amplitude $\gamma+A \rightarrow V+A$ is the sum of Coulomb (Fig. 1), $M_{C}$, and strong (Fig. 2), $M_{s}$, production amplitudes.

As we argued, the Coulomb amplitude can be evaluated to the lowest order in QED perturbation theory, i.e. the two-photon exchange approximation. Because the typical impact parameters which contribute to the Coulomb amplitude are much larger than the size of the vector meson, $|\vec{b}| \sim 1 / \Delta=2 E_{\gamma} / m_{V}^{2} \gg R_{V}$, the principal quantity is an imaginary part of an amplitude, $f(\vec{r}, \vec{q})$, of a nearly forward scattering of the $q \bar{q}$ electric dipole in the Coulomb field of a nucleus. With some important modifications for the effect of the finite longitudinal momentum transfer, which amounts to an effective screening of the Coulomb potential, the amplitude $f(\vec{r}, \vec{q})$ is related to the electric dipole scattering cross section $\sigma_{C}(\vec{r})$, where $\vec{r}$ is the $q \bar{q}$ separation in the two-dimensional impact parameter space. The latter enters, for instance, the calculation of a scattering of atoms in a Coulomb field, the case of muonium (or pionium [?]) is the most relevant one because the size of muonium is much smaller than the radius of an atom and nucleus acts predominantly as a pointlike charge. If we define $f(\vec{r}, \vec{q})$ for the electric dipole made of the particles of charge \pm 1 , then the imaginary part of the Coulomb amplitude can be cast in the form

$$
M_{C}(\vec{q})=C_{C}(V)\left\langle V|f(\vec{r}, \vec{q})| \gamma>=\frac{e}{F_{V}}\langle V|f(\vec{r}, \vec{q})| V\rangle\right.
$$

where the charge-isotopic factors equal

$$
\begin{array}{r}
C_{C}(\rho)=\frac{1}{\sqrt{2}}\left(e_{u}^{3}+e_{d}^{3}\right)=\frac{1}{3 \sqrt{2}} \\
C_{C}(\omega)=\frac{1}{\sqrt{2}}\left(e_{u}^{3}-e_{d}^{3}\right)=\frac{7}{27 \sqrt{2}} \\
C_{C}(\phi)=e_{s}^{3}=\frac{1}{27}
\end{array}
$$

In (4) we invoked vector dominance model (for an extensive review and references see [8]) and $F_{V}$ are related to the standard vector dominance 
constants $f_{V}$ as

$$
\begin{array}{r}
\frac{1}{F_{\rho}}=\frac{1}{f_{V}}\left(e_{u}^{2}-e_{u} e_{d}+e_{d}^{2}\right)=\frac{7}{9 f_{\rho}} \\
\frac{1}{F_{\omega}}=\frac{1}{f_{V}}\left(e_{u}^{2}+e_{u} e_{d}+e_{d}^{2}\right)=\frac{1}{3 f_{\omega}} \\
\frac{1}{F_{\phi}}=\frac{1}{f_{\phi}} e_{s}^{2}=\frac{1}{9 f_{\phi}}
\end{array}
$$

The amplitude (4) is normalized so that

$$
\frac{d \sigma}{d q^{2}}=\frac{|M|^{2}}{16 \pi}
$$

In the calculation of the amplitude $f(\vec{r}, \vec{q})$ a care must be taken of the longitudinal momentum transfer. Interaction of the incoming photon with the first nuclear photon puts the quark-antiquark pair on mass shell, and the exchanged photon has a longitudinal momentum

$$
\Delta_{1}=\frac{M^{2}}{2 E_{\gamma}}
$$

where $M$ is the invariant mass of the $q \bar{q}$ pair. The second photon has a longitudinal momentum

$$
\Delta_{2}=\frac{M_{V}^{2}-M^{2}}{2 E_{\gamma}} .
$$

In the nonrelativistic quark model $M \sim M_{V}$ and $\left|\Delta_{2}\right| \ll \Delta_{1}$. This hierarchy is a good starting approximation for light mesons as well. In what follows we put $\Delta_{1}=\Delta=m_{V}^{2} / 2 E_{\gamma}$ and $\Delta_{2}=0$. For high energy photons the size of the vector meson can be neglected compared to $1 / \Delta$ and $f(\vec{r}, \vec{q})$ can be cast in the form,

$$
\begin{array}{r}
f(\vec{r}, \vec{q})=\left(Z \alpha_{e m}\right)^{2} \vec{r}^{2} \int \frac{d^{2} \vec{k}\left(\vec{k}-\frac{1}{2} \vec{q}, \vec{k}+\frac{1}{2} \vec{q}\right) F_{A}\left(\vec{k}-\frac{1}{2} \vec{q}\right) F_{A}\left(\vec{k}+\frac{1}{2} \vec{q}\right)}{\left[\left(\vec{k}-\frac{1}{2} \vec{q}\right)^{2}+\Delta^{2}\right]\left(\vec{k}+\frac{1}{2} \vec{q}\right)^{2}} \\
=\pi\left(Z \alpha_{e m}\right)^{2} \vec{r}^{2} \ln \frac{1}{\left(q^{2}+\Delta^{2}\right)\left\langle R_{A}^{2}\right\rangle_{c h}},
\end{array}
$$

which clearly exhibits the logarithmic singularity of the Coulomb amplitude as a function of $\vec{q}^{2}$. Here $\left\langle\vec{R}_{A}^{2}\right\rangle_{c h}$ is the charge radius of the nucleus squared, and hereafter the capital $\vec{R}$ stands for the 3 -dimensional radius-vector. The 
substitution of (10) into (4) gives

$$
\begin{aligned}
M_{C}(\vec{q}) & =\frac{\pi e\left(Z \alpha_{e m}\right)^{2}}{F_{V}}\left\langle V\left|\vec{r}^{2}\right| V\right\rangle \ln \frac{6}{\left(q^{2}+\Delta^{2}\right)\left\langle R_{A}^{2}\right\rangle_{c h}} \\
& =\frac{8 \pi e\left(Z \alpha_{e m}\right)^{2}}{3 F_{V}}\left\langle\vec{R}_{V}^{2}\right\rangle_{c h} \ln \frac{6}{\left(q^{2}+\Delta^{2}\right)\left\langle R_{A}^{2}\right\rangle_{c h}}
\end{aligned}
$$

where in the case of the $\rho^{ \pm}$-meson $\left\langle\vec{R}_{V}^{2}\right\rangle_{\text {ch }}$ is the conventionally defined 3dimensional charge radius mean squared. Consequently, the experimental isolation of the ultraperipheral Coulomb production amplitude would give a unique possibility to measure the radius of the vector meson squared.

\section{The amplitude of photoproduction via strong interaction}

Within the QCD color dipole framework, one needs first to calculate the amplitude of interaction of the $q \bar{q}$ color dipole with the target, and then use the same formalism as outlined above for electric dipoles [9]. However, for the illustration purposes it is more straightforward to invoke the vector dominance model by which

$$
M_{s}(\vec{q})=\frac{e}{f_{V}} M_{V A}(\vec{q})
$$

Anyway, the real photoproduction of vector mesons is a soft process and $M_{s}(\vec{q})$ can not be reliably calculated in the pQCD framework. The vector meson-nucleus scattering amplitude can be evaluated in the standard Glauber approximation. For a simple as yet sufficiently accurate Gaussian parameterization for a nuclear matter density,

$$
n_{A}(r)=n_{0} \exp \left(-\frac{r^{2}}{R_{A}^{2}}\right)
$$

one finds the familiar expression

$$
\begin{aligned}
M_{V A}(\vec{q})= & A \sigma_{V N} \exp \left(\frac{R_{A}^{2} q^{2}}{4 A}\right) \sum_{n=1}^{A} \frac{(A-1) !}{n \cdot n !(A-n) !} \\
& \times\left[-\frac{\sigma(V N)}{2 \pi\left(R_{A}^{2}+2 B_{N}\right)}\right]^{n-1} \exp \left(-\frac{\left(R_{A}^{2}+2 B_{N}\right) q^{2}}{4 n}\right),
\end{aligned}
$$

where $B_{N}$ is the diffraction slope of the $V N$ elastic scattering. In (14) we neglected the small real part of the VN scattering amplitude. The total 
cross section of the vector meson interaction with nucleon $\sigma(V N)$ can be obtained from the relation

$$
\sigma(V N)=\frac{\sigma\left(\pi^{+} N\right)+\sigma\left(\pi^{-} N\right)}{2} .
$$

For the estimation purposes, one can neglect $B_{N}$ compared to $R_{A}^{2}$ and use the simple parameterization

$$
M_{V A}(\vec{q}) \approx \sigma(V N) A^{\alpha} \exp \left[-\frac{1}{2} B_{A} \vec{q}^{2}\right]
$$

where for the $\rho$ and $\omega$ the exponent $\alpha \approx 0.8$ and to the crude approximation $B_{A} \approx \frac{1}{3}\left\langle R_{A}^{2}\right\rangle_{c h}$.

\section{Is the Coulomb correction observable?}

The Coulomb correction to the differential cross section is small,

$$
\begin{array}{r}
\frac{2 M_{C}}{M_{s}}=\eta_{C} \ln \frac{6}{\left(\vec{q}^{2}+\Delta^{2}\right)\left\langle\vec{R}_{A}^{2}\right\rangle_{c h}} \\
=\frac{16 \pi\left(Z \alpha_{e m}\right)^{2} f_{V}}{3 F_{V} A^{\alpha}} \cdot \frac{\left\langle\vec{R}_{V}^{2}\right\rangle_{c h}}{\sigma(V M)} \cdot \ln \frac{6}{\left(\vec{q}^{2}+\Delta^{2}\right)\left\langle\vec{R}_{A}^{2}\right\rangle_{c h}} .
\end{array}
$$

Take the photoproduction of the $\rho$ meson off the lead target, for which $\left\langle\vec{R}_{A}^{2}\right\rangle_{c h} \approx 25 \mathrm{f}^{2}$. The nonrelativistic quark model suggests $\left\langle\vec{R}_{V}^{2}\right\rangle_{c h} \approx\left\langle\vec{R}_{\pi}^{2}\right\rangle_{c h} \approx$ $0.4 \mathrm{f}^{2}$ and $\sigma(\rho N) \sim 30 \mathrm{mb}$. For $E_{\gamma}=10 \mathrm{GeV}$ the logarithmic factor is of the order of 2 , so that the Coulomb correction is negligible small, $\sim 1.5 \%$.

The logarithmic singularity of $2 M_{C}(\vec{q})$ makes the Coulomb correction much more noticeable in the diffraction slope

$$
B=2 \frac{d \ln M}{d \vec{q}^{2}}
$$

Using the results $(15),(17),(18),(22)$ one obtains for the slope of the full amplitude

$$
B=B_{A}+\Delta B_{C}=B_{A}+\eta_{C} \frac{1}{\vec{q}^{2}+\Delta^{2}}=B_{A}+\frac{4 \eta_{C} E_{\gamma}^{2}}{m_{\rho}^{4}} \cdot \frac{\Delta^{2}}{\vec{q}^{2}+\Delta^{2}} .
$$

In order to emphasize the importance of the Coulomb correction to the diffraction slope, $\Delta B_{C}$, start with the exactly forward, $\vec{q}^{2}=0$, photoproduction off the lead target. Here teh Coulomb correction rises from $\Delta B_{C} \sim 20 \mathrm{GeV}^{-2}$ at $E_{\gamma}=10 \mathrm{GeV}$ to a dramatically large $\Delta B_{C} \sim 2000$ $\mathrm{GeV}^{-2}$ to be compared to $B_{A} \sim 200 \mathrm{GeV}^{-} 2$ at $E_{\gamma}=100 \mathrm{GeV}$. Of course, 
this dramatic rise is confined to a region of ultrasmall momentum transfers, from $|\vec{q}| \sim \Delta \approx 2.5 \mathrm{MeV} / \mathrm{c}$ at $E_{\gamma}=100 \mathrm{GeV}$, which are perhaps beyond the experimental reach, to a more realistic $|\vec{q}| \sim \Delta \approx 25 \mathrm{MeV} / \mathrm{c}$ at $E_{\gamma}=10 \mathrm{GeV}$. Now notice, that while the prefactor $\eta_{C}$ does not depend on our approximations for $\Delta_{1,2}$, the exact $\vec{q}^{2}$ dependence, and the magnitude at $\vec{q}^{2}=0$, of the $\Delta B_{C}$, do explicitly depend on $\Delta_{1,2}$. Consequntly, for the accurate determination of the size of the $\rho$-meson one must concentrate on the Coulomb correction $\Delta B_{C}$ at $\vec{q}^{2}>\Delta^{2}$, where the $\vec{q}^{2}$ dependence is insensitive to $\Delta_{1,2}$.

\section{Summary and conclusions}

We pointed out a new, ultraperipheral Coulomb, mechanims for the photoproduction of vector mesons off nuclei at ultrasmall momentum transfer. The Coulomb contribution to the photoproduction amplitude has a singular, logarithmic, dependence on the momentum transfer squared, and is proportional to the mean radius of the vector veson squared. This strongly energy dependent singular contribution entails a steep dependence of the diffraction slope on $\vec{q}^{2}$. The very specific dependence of the Coulomb correction $\Delta B_{C}$ on the photon energy, the momentum transfer squared $\vec{q}^{2}$, the target charge and mass number makes the experimental evaluation of the size of the $\rho$ meson quite plausible. The work of I.I. and S.G. has been partly supported by the grants INTAS 00-0036 and INTAS 97-30494.

\section{References}

1. Primakoff,H.(1951) Photoproduction of neutral mesons in nuclear electric fields and the mean life of the neutral mesons, Phys. Rev. 81,899

2. Landsberg,L.G.(1985) Electromagnetic decays of light mesons, Phys. Reports 128, 303

3. Gasparian,A. et al.(1998) A precision measurement of the neutral pion lifetime via the Primakoff effect, TJNAF Proposal PR-98-105

4. Pomeranchuk,I.Y.and Shmushkevich,I.M.(1961) On processes in the interaction of $\gamma$-quanta with unstable particles, Nucl. Phys. 23,452

5. Schwiete,G. (2000) The higher order corrections to Primakoff effect, Acta Phys. Polon. B31, 2437 ; Diploma Thesis, Institut f. Kernphysik, Forschungszentrum Jülich \& University of Bonn, 2000.

6. Review of Particle Properties (1998) The European Phys.J.,3,1

7. Gevorkyan,S.R.,Tarasov,A.V. and Voskresenskaya,O.O.(1998) Total Cross Sections for the Interaction of Hydrogen-like Atoms with Atoms of a Medium,Physics of Atomic Nuclei 61,1507

8. Bauer,T.H.,Spital,R.D.,Yennie,D.R. and Pipkin,F.M.(1978) The hadronic properties of the photon in high energy interactions, Rev. Mod. Phys. 50, 261

9. Kopeliovich,B.Z., Nemchik,J., Nikolaev,N.N. and Zakharov,B.G. (1994) Scanning the BFKL pomeron in elastic production of vector mesons at HERA, Phys. Lett. B341, 228 